\title{
FACTORING SOBOLEV INEQUALITIES THROUGH CLASSES OF FUNCTIONS
}

\author{
DAVID ALONSO-GUTIÉRREZ, JESÚS BASTERO, AND JULIO BERNUÉS
}

(Communicated by Marius Junge)

\begin{abstract}
We recall two approaches to recent improvements of the classical Sobolev inequality. The first one follows the point of view of Real Analysis, while the second one relies on tools from Convex Geometry. In this paper we prove a (sharp) connection between them.
\end{abstract}

\section{INTRODUCTION AND NOTATION}

The classical Sobolev inequality states that for $1 \leq p<n$ and $\frac{1}{q}=\frac{1}{p}-\frac{1}{n}$, there exists a constant $C_{p, n}>0$ such that for every $f: \mathbb{R}^{n} \rightarrow \mathbb{R}$ in the Sobolev space $W^{1, p}\left(\mathbb{R}^{n}\right)$,

$$
\|\nabla f\|_{p} \geq C_{p, n}\|f\|_{q}
$$

where $\|\cdot\|_{q}$ denotes the $L_{q}-$ norm of the Euclidean norm of functions and $\nabla f$ is the gradient of $f$.

The best constant in the case $p=1(q=n /(n-1))$ was obtained by H. Federer, W. Fleming [8] and independently by V. Maz'ja [18], [19]. They proved that $C_{1, n}=$ $n \omega_{n}^{\frac{1}{n}}$, where $\omega_{n}$ denotes the Lebesgue measure of the Euclidean unit ball in $\mathbb{R}^{n}$, and showed that this fact is equivalent to the isoperimetric inequality (see for instance [5] for a survey). For the other values of $1<p<n$, Aubin and Talenti got the best constants [1, 28. See also the recent approaches in [2] and [7.

We point out that one key step in the classical proofs of (1.1) is the use of the Polya-Szegö rearrangement inequality (see [26]),

$$
\|\nabla f\|_{p} \geq\left\|\nabla f^{\circ}\right\|_{p}, \quad \quad p \geq 1,
$$

where $f^{\circ}(x):=f^{*}\left(\omega_{n}|x|^{n}\right)$ is a radial extension to $\mathbb{R}^{n}$ of the decreasing rearrangement of $f, f^{*}(t)=\inf \left\{\lambda>0:|\{|f|>\lambda\}|_{n} \leq t\right\}, t \geq 0,|\cdot|$ is the Euclidean distance in $\mathbb{R}^{n}$ and $|\cdot|_{n}$ is the Lebesgue measure on the (suitable) $n$-dimensional space. $f^{\circ}$ has the same distribution function as $f$ and $f^{*}$. It is called the symmetric Schwarz rearrangement of $f$.

For $p=n$, the inequality with $q=\infty$ is not true. In the sixties, Trudinger 31] and Moser 23. proposed an Orlicz space, $\mathcal{M T}(\Omega)$, of functions defined on open domains $\Omega \subset \mathbb{R}^{n}$ with $|\Omega|_{n}<\infty$ and showed the continuous inclusion $W_{0}^{1, n}(\Omega) \hookrightarrow$ $\mathcal{M T}(\Omega)$, where $W_{0}^{1, n}(\Omega)$ is the closure of the space of $\mathcal{C}^{1}$ functions of compact

Received by the editors October 20, 2010 and, in revised form, April 15, 2011.

2010 Mathematics Subject Classification. Primary 46E35; Secondary 46E30, 26D10, 52A40.

Key words and phrases. Sobolev inequality, sharp constants, affine isoperimetric inequalities.

The three authors were partially supported by MCYT Grant(Spain) MTM2010-16679 and DGA E-64. 
support, $\mathcal{C}_{00}^{1}(\Omega)$, in the Sobolev space $W^{1, n}(\Omega)$. More precisely, they proved that there exists $C_{n}>0$ such that for all $f \in W_{0}^{1, n}(\Omega)$,

$$
\|\nabla f\|_{n} \geq C_{n}\|f\|_{\mathcal{M} \mathcal{T}}
$$

and the constant (depending on $|\Omega|_{n}$ ) is sharp.

In the late seventies, Hansson [12] and Brézis-Wainger [4] improved the target space in the inclusion above. They introduced a rearrangement-invariant function space, $H_{n}(\Omega)$, such that $W_{0}^{1, n}(\Omega) \hookrightarrow H_{n}(\Omega) \hookrightarrow \mathcal{M T}(\Omega)$. Moreover, $H_{n}(\Omega)$ was proved to be the optimal target space in the class of rearrangement-invariant spaces. Equivalently, they obtained an inequality of the form $\|\nabla f\|_{n} \geq c_{n}\|f\|_{H_{n}} \geq$ $c_{n}^{\prime}\|f\|_{\mathcal{M T}}$ for some constants $c_{n}, c_{n}^{\prime}>0$ (depending on $|\Omega|_{n}$ ).

Tartar [30, Malý-Pick [20] and Bastero-Milman-Ruiz [3] (see also [13]) refined those estimates using classes of functions as follows: For $1 \leq p<\infty$ denote

$$
\mathcal{A}_{\infty, p}\left(\mathbb{R}^{n}\right)=\left\{f:\|f\|_{\infty, p}=\left(\int_{0}^{\infty}\left(f^{* *}(t)-f^{*}(t)\right)^{p} \frac{d t}{t^{p / n}}\right)^{1 / p}<\infty\right\},
$$

where $f^{* *}$ is the Hardy transform of $f^{*}$ defined by $f^{* *}(t)=\frac{1}{t} \int_{0}^{t} f^{*}(s) d s . W^{1, p}\left(\mathbb{R}^{n}\right)$. Then for all $f \in W_{0}^{1, n}(\Omega)$,

$$
\|\nabla f\|_{n} \geq(n-1) \omega_{n}^{\frac{1}{n}}\|f\|_{\infty, n} \geq c_{n}^{\prime \prime}\|f\|_{H_{n}}
$$

for some $c_{n}^{\prime \prime}>0$ (depending on $|\Omega|_{n}$ ). Observe that the constant in the first inequality depends neither on the measure of $\Omega$ nor on the support of $f$.

Once one considered classes of functions instead of vector spaces, Sobolev type inequalites could be extended further. At this point we recall the well-known fact that $W_{0}^{1, p}\left(\mathbb{R}^{n}\right)=W^{1, p}\left(\mathbb{R}^{n}\right)$, [19]. In [21] the authors proved

$$
\|\nabla f\|_{p} \geq c_{n, p}\|f\|_{\infty, p} \geq c_{n, p}^{\prime}\|f\|_{q}, \quad \forall f \in W^{1, p}\left(\mathbb{R}^{n}\right), \quad 1 \leq p<n
$$

and some constants $c_{n, p}, c_{n, p}^{\prime}>0$.

We now move on to a different philosophy. We start by recalling the so-called Petty projection inequality, stated in 25 . for convex bodies and extended by Zhang [32] to compact subsets $K \subset \mathbb{R}^{n}$,

$$
\frac{n \omega_{n}}{\omega_{n-1}}\left(\int_{S^{n-1}} \frac{d u}{\left|P_{u^{\perp}}(K)\right|_{n-1}^{n}}\right)^{-\frac{1}{n}} \geq n \omega_{n}^{1 / n}|K|_{n^{\frac{n-1}{n}}}
$$

where $P_{u^{\perp}}$ is the orthogonal projection onto the hyperplane $u^{\perp}$ and $d u$ is the normalized Haar probability on the unit sphere $S^{n-1}$. The Petty projection inequality directly implies the isoperimetric inequality.

In 1999 Zhang 32 (see also 11 and the references therein) introduced a new class of functions

$\mathcal{E}_{p}\left(\mathbb{R}^{n}\right)=\left\{f \in W^{1, p}\left(\mathbb{R}^{n}\right) ; \mathcal{E}_{p}(f):=\frac{1}{I_{p}}\left(\int_{S^{n-1}}\left\|D_{u} f\right\|_{p}^{-n} d u\right)^{-\frac{1}{n}}<\infty\right\}, \quad p \geq 1$,

where $D_{u} f(x):=\langle\nabla f(x), u\rangle$ and $I_{p}^{p}:=\int_{S^{n-1}}\left|u_{1}\right|^{p} d u$ is a normalization constant so that $\mathcal{E}_{p}\left(f^{\circ}\right)=\left\|\nabla f^{\circ}\right\|_{p}$. The expression $\mathcal{E}_{p}(f)$ is an energy integral having applications in information theory. It is invariant under transformations of $\mathbb{R}^{n}$ of the 
form $x \rightarrow x_{0}+A x, x_{0} \in \mathbb{R}^{n}, A \in S L(n)$, [17. Moreover, by Jensen's inequality and Fubini's theorem the following relation holds:

$$
\mathcal{E}_{p}(f)=\frac{1}{I_{p}}\left(\int_{S^{n-1}}\left\|D_{u} f\right\|_{p}^{-n} d u\right)^{-1 / n} \leq \frac{1}{I_{p}}\left(\int_{S^{n-1}}\left\|D_{u} f\right\|_{p}^{p} d u\right)^{1 / p}=\|\nabla f\|_{p} .
$$

The following remarkable inequality,

$$
\mathcal{E}_{p}(f) \geq \mathcal{E}_{p}\left(f^{\circ}\right), \quad 1 \leq p<\infty,
$$

was proved in a series of papers: Zhang [32] initiated the approach by showing that his extension of the Petty projection inequality (1.5) is actually equivalent to (1.6) for $p=1$. The general case was proved via the $L_{p}$-Brunn-Minkowski theory in [16], [17, 6]. The invariance of $\mathcal{E}_{p}(f)$ implies, by homogeneity, that (1.6) is affine-invariant, i.e., invariant under transformations of $\mathbb{R}^{n}$ of the form $x \rightarrow x_{0}+A x, x_{0} \in \mathbb{R}^{n}, A \in G L(n)$.

The inequality (1.6) is stronger than the Polya-Szegö rearrangement inequality (1.2) and thus it yields to a new proof of Sobolev's inequality,

$$
\|\nabla f\|_{p} \geq \mathcal{E}_{p}(f) \geq \mathcal{E}_{p}\left(f^{\circ}\right) \geq C_{n, p}\|f\|_{q}, \quad 1 \leq p<n .
$$

See [32, 17] for such a proof of (1.1) with sharp constants. We point out the fact that $\mathcal{E}_{p}(f) \geq C_{n, p}\|f\|_{q}$ is affine-invariant while Sobolev's inequality $\|\nabla f\|_{p} \geq$ $C_{n, p}\|f\|_{q}$ is invariant only under rigid motions.

In [9], 10], 11], the authors investigated the space $\mathcal{E}_{p}^{+}\left(\mathbb{R}^{n}\right)$ defined analogously as before by

$$
\mathcal{E}_{p}^{+}(f):=\frac{2^{1 / p}}{I_{p}}\left(\int_{S^{n-1}}\left\|D_{u}^{+} f\right\|_{p}^{-n} d u\right)^{-\frac{1}{n}},
$$

where $D_{u}^{+} f(x):=\max \{\langle\nabla f(x), u\rangle, 0\}$, and proved

$$
\mathcal{E}_{p}(f) \geq \mathcal{E}_{p}^{+}(f) \geq \mathcal{E}_{p}^{+}\left(f^{\circ}\right)=\mathcal{E}_{p}\left(f^{\circ}\right), \quad p \geq 1,
$$

which refined (1.7) for $1 \leq p<n$.

In the case $p \geq n$, affine-invariant inequalities of Sobolev type were studied in [6], 10] and [11] with the hypothesis of $f$ having support of finite measure. In the limiting case $p=n$, they proved the sharp inequality

$$
\|\nabla f\|_{n} \geq \mathcal{E}_{n}(f) \geq \mathcal{E}_{n}^{+}(f) \geq C_{n}\|f\|_{\mathcal{M T}},
$$

while for $p>n$,

$$
\mathcal{E}_{p}(f) \geq \mathcal{E}_{p}^{+}(f) \geq\left(\frac{p^{\prime}}{|q|}\right)^{\frac{1}{p^{\prime}}} n \omega_{n}^{1 / n}\|f\|_{\infty}|\operatorname{supp} f|_{n}^{1 / q}, \quad \frac{1}{p}+\frac{1}{p^{\prime}}=1,
$$

where the constant depending on the size of the support of $f$ is sharp (take $f^{*}(t)=$ $\left.\left(1-t^{-p^{\prime} / q}\right) \chi_{[0,1]}(t)\right)$.

In conclusion, the first approach so described looked for improvements of the right hand side of (1.1), while the second approach concerned the left hand side of (1.1). In this paper we link these two approaches and show that for all $1 \leq p<\infty$ and $\frac{1}{q}:=\frac{1}{p}-\frac{1}{n}$,

$$
\mathcal{E}_{p}^{+}(f) \geq\left(1-\frac{1}{q}\right) n \omega_{n}^{1 / n}\|f\|_{\infty, p} \quad \forall f \in W^{1, p}\left(\mathbb{R}^{n}\right)
$$

(see Theorem 2.1) where the constant is sharp. As a consequence, for $1 \leq p<n$ it gives the right constant in the first inequality in (1.4) and enables us to connect 
(1.7), (1.8) and (1.4). For $p=n$, Theorem 2.1] and its Corollary 2.2 connect (1.3) and (1.9), improving the first inequality in (1.3). In the case $p>n$ it links the first inequality in (1.4) and (1.7) (thanks to (1.8) and to the fact that they are also valid for $p>n$ ). Moreover, in Proposition 2.4, we see how it yields to lower estimates for $\mathcal{E}_{p}^{+}(f)$ better than (1.10).

In the third section we include a proof of the inequalities (1.6) and (1.8) which directly derives from Zhang's extension of the Petty projection inequality, paying the penalty of an extra constant $\frac{I_{p}}{I_{1}}$ (which is independent of the dimension $n$ ). No use of the $L_{p}$-Brunn-Minkoswki theory and polytope approximation appearing in the papers [16], [17, 6] and [10] is made.

\section{The RESUlts}

The first result is the correct relation between $\mathcal{E}_{p}^{+}(f)$ and $\|f\|_{\infty, p}$.

Theorem 2.1. Let $1 \leq p<\infty$ and $\frac{1}{q}=\frac{1}{p}-\frac{1}{n}, q \in(-\infty,-n) \cup\left[\frac{n}{n-1}, \infty\right]$. Then

$$
\mathcal{E}_{p}^{+}(f) \geq\left(1-\frac{1}{q}\right) n \omega_{n}^{1 / n}\|f\|_{\infty, p} \quad \forall f \in W^{1, p}\left(\mathbb{R}^{n}\right) .
$$

Moreover the constant is sharp.

Proof. Taking (1.8) into account it is enough to see that for any compactly supported $C^{1}$ function $f: \mathbb{R}^{n} \rightarrow \mathbb{R}$,

$$
\mathcal{E}_{p}^{+}\left(f^{\circ}\right)=\frac{2^{1 / p}}{I_{p}}\left(\int_{S^{n-1}}\left\|D_{u}^{+} f^{\circ}\right\|_{p}^{-n} d u\right)^{-1 / n} \geq\left(1-\frac{1}{q}\right) n \omega_{n}^{1 / n}\|f\|_{\infty, p} .
$$

Now, $f^{\circ}$ is Lipschitz and $f^{*}$ locally Lipschitz and $f^{* \prime}$ integrable (see for instance [13] and 24]). Therefore since

$f^{* *}(t)-f^{*}(t)=\frac{1}{t} \int_{0}^{t}\left(f^{*}(s)-f^{*}(t)\right) d s=\frac{1}{t} \int_{0}^{t} \int_{s}^{t}-f^{* \prime}(u) d u d s=\frac{1}{t} \int_{0}^{t} s\left|f^{* \prime}(s)\right| d s$

we have

$$
\left\|f^{\circ}\right\|_{\infty, p}^{p}=\|f\|_{\infty, p}^{p}=\int_{0}^{\infty}\left(f^{* *}(t)-f^{*}(t)\right)^{p} \frac{d t}{t^{p / n}}=\int_{0}^{\infty}\left(\frac{1}{t} \int_{0}^{t} s\left|f^{* \prime}(s)\right| d s\right)^{p} \frac{d t}{t^{p / n}} .
$$

Apply Hardy's inequality $\int_{0}^{\infty}\left(\frac{1}{t} \int_{0}^{t} g(s) d s\right)^{p} \frac{d t}{t^{p / n}} \leq\left(\frac{p}{p+\frac{p}{n}-1}\right)^{p} \int_{0}^{\infty} g(s)^{p} \frac{d s}{s^{p / n}}$ to $g(s)=s\left|f^{* \prime}(s)\right|$ (see [19] for a reference on Hardy's inequalities with weights) to obtain

$$
\left\|f^{\circ}\right\|_{\infty, p}^{p} \leq\left(\frac{1}{1-\frac{1}{q}}\right)^{p} \int_{0}^{\infty} s^{\frac{(n-1) p}{n}}\left|f^{* \prime}(s)\right|^{p} d s .
$$

On the other hand, by definition of $f^{\circ}(x)$,

$$
\left\langle\nabla f^{\circ}(x), u\right\rangle_{+}=n \omega_{n}|x|^{n-1}\left|f^{* \prime}\left(\omega_{n}|x|^{n}\right)\right|\left\langle\frac{-x}{|x|}, u\right\rangle_{+}
$$

and so by polar integration $x=r \theta$ and the change of variables $s=\omega_{n} r^{n}$,

$$
\left\|D_{u}^{+} f^{\circ}\right\|_{p}^{p}=\int_{\mathbb{R}^{n}}\left\langle\nabla f^{\circ}(x), u\right\rangle_{+}^{p} d x=\frac{1}{2} I_{p}^{p}\left(n \omega_{n}^{1 / n}\right)^{p} \int_{0}^{\infty} s^{\frac{(n-1) p}{n}}\left|f^{* \prime}(s)\right|^{p} d s
$$

and the result follows. 
In order to see that the constant is sharp, consider truncations of the function $f^{*}(t)=t^{-1 / q}$ whenever $p<n, f^{*}(t)=\log (1 / t)$, for $p=n$, and $f^{*}(t)=(1-$ $\left.t^{-1 / q}\right) \chi_{[0,1]}$ whenever $p>n$.

Straightforward computations show that $f^{*}$ and therefore $\|f\|_{\infty, p}$ are invariant under transformations of $\mathbb{R}^{n}, x \rightarrow x_{0}+A x, x_{0} \in \mathbb{R}^{n}, A \in S L(n)$. Consequently, the inequality in Theorem 2.1 is affine-invariant.

Corollary 2.2. Let $1 \leq p<\infty$. For any $f \in W^{1, p}\left(\mathbb{R}^{n}\right)$,

$$
\|\nabla f\|_{p} \geq n \omega_{n}^{1 / n}\left(1-\frac{1}{q}\right)\|f\|_{\infty, p}
$$

In particular, for $p=n$ we have $\|\nabla f\|_{n} \geq n \omega_{n}^{1 / n}\|f\|_{\infty, n}$.

Remark 2.3. The result refines the inequalities (1.4), (1.3) and (1.9).

Proof. It follows from the previous theorem and the facts stated in the introduction that

$$
\left(1-\frac{1}{q}\right) n \omega_{n}^{1 / n}\|f\|_{\infty, p} \leq \mathcal{E}_{p}^{+}\left(f^{\circ}\right) \leq \mathcal{E}_{p}\left(f^{\circ}\right) \leq \mathcal{E}_{p}(f) \leq\|\nabla f\|_{p} .
$$

We pass to the case $p>n$. As we said in the introduction we shall see how Theorem 2.1 provides better estimates than inequality (1.10).

Proposition 2.4. Let $p>n, \frac{1}{q}=\frac{1}{p}-\frac{1}{n}$ and $f$ a compactly supported $C^{1}$ function. Then,

$$
\sup _{t>0}\left\{\left(\|f\|_{\infty}-f^{*}(t)\right) t^{1 / q}\right\} \leq \alpha_{n, p}\|f\|_{\infty, p}
$$

for some $\alpha_{n, p}>0$ (independent of the support of $f$ ).

Remark 2.5. The proof gives $\alpha_{p, n}=\left(\left(p\left(1-\frac{1}{q}\right)\right)^{p^{\prime} / p}+\frac{|q|}{p^{\prime}}\right)^{\frac{1}{p^{\prime}}}$.

Proof. For any $t>0$ we have

$$
\begin{aligned}
\|f\|_{\infty} & -f^{*}(t)=f^{* *}(0)-f^{* *}(t)+f^{* *}(t)-f^{*}(t)=\int_{0}^{t}-f^{* * \prime}(u) d u+f^{* *}(t)-f^{*}(t) \\
& =\int_{0}^{t} \frac{f^{* *}(u)-f^{*}(u)}{u} d u+f^{* *}(t)-f^{*}(t) \\
& =\int_{0}^{t} \frac{f^{* *}(u)-f^{*}(u)}{u} d u\left(\frac{p^{\prime}}{|q|}\right)^{\frac{1}{p^{\prime}}}\left(\frac{|q|}{p^{\prime}}\right)^{\frac{1}{p^{\prime}}}+\frac{f^{* *}(t)-f^{*}(t)}{\left(p\left(1-\frac{1}{q}\right)\right)^{\frac{1}{p}}}\left(p\left(1-\frac{1}{q}\right)\right)^{\frac{1}{p}} .
\end{aligned}
$$

By Hölder's inequality, the latter expression is bounded from above by

$$
\alpha_{p, n}\left(\left(\int_{0}^{t} \frac{f^{* *}(u)-f^{*}(u)}{u} d u\right)^{p}\left(\frac{p^{\prime}}{|q|}\right)^{\frac{p}{p^{\prime}}}+\frac{\left(f^{* *}(t)-f^{*}(t)\right)^{p}}{p\left(1-\frac{1}{q}\right)}\right)^{\frac{1}{p}} .
$$

Now, on the one hand, for any $s>t>0$ we have, after integrating by parts,

$$
f^{* *}(s)-f^{*}(s)=\frac{1}{s} \int_{0}^{s} u\left|f^{* \prime}(u)\right| d u \geq \frac{1}{s} \int_{0}^{t} u\left|f^{* \prime}(u)\right| d u=\frac{t}{s}\left(f^{* *}(t)-f^{*}(t)\right) .
$$


Hence

$$
\int_{t}^{\infty}\left(f^{* *}(s)-f^{*}(s)\right)^{p} s^{-p / n} d s \geq t^{p}\left(f^{* *}(t)-f^{*}(t)\right)^{p} \int_{t}^{\infty} \frac{d s}{s^{p+\frac{p}{n}}}
$$

and consequently

$$
\frac{\left(f^{* *}(t)-f^{*}(t)\right)^{p}}{p\left(1-\frac{1}{q}\right)} \leq t^{-\frac{p}{q}} \int_{t}^{\infty}\left(f^{* *}(s)-f^{*}(s)\right)^{p} s^{-p / n} d s .
$$

On the other hand, by Hölder's inequality and since $p>n$,

$$
\int_{0}^{t} \frac{f^{* *}(s)-f^{*}(s)}{s} \leq\left(\int_{0}^{t} s^{\left(\frac{1}{n}-1\right) p^{\prime}} d s\right)^{1 / p^{\prime}}\left(\int_{0}^{t}\left(f^{* *}(s)-f^{*}(s)\right)^{p} s^{-p / n} d s\right)^{1 / p}
$$

which implies

$$
\left(\int_{0}^{t} \frac{f^{* *}(s)-f^{*}(s)}{s}\right)^{p}\left(\frac{p^{\prime}}{|q|}\right)^{\frac{p}{p^{\prime}}} t^{\frac{p}{q}} \leq \int_{0}^{t}\left(f^{* *}(s)-f^{*}(s)\right)^{p} s^{-p / n} d s .
$$

Thus, for any $t>0$ we have

$$
t^{\frac{1}{q}}\left(\|f\|_{\infty}-f^{*}(t)\right) \leq \alpha_{p, n}\|f\|_{\infty, p},
$$

which finishes the proof.

Remark 2.6. $f^{*}\left(|\operatorname{supp} f|_{n}\right)=0$ implies $\|f\|_{\infty}|\operatorname{supp} f|_{n}^{1 / q} \leq \sup _{t>0}\left\{\left(\|f\|_{\infty}-f^{*}(t)\right) t^{1 / q}\right\}$, and so Proposition 2.4 shows that Theorem 2.1 is, up to a constant, better than (1.10). The example $f^{*}(t)=\left(1-t^{-1 / q}\right) \chi_{[0,1]}(t)$ satisfies $\sup _{t>0}\left\{\left(\|f\|_{\infty}-f^{*}(t)\right) t^{1 / q}\right\}=$ 1 while $\|f\|_{\infty, p}=\infty$.

\section{A Simplified APPRoach to AFFine Sobolev inequalities}

In this part we will show a direct way to deduce the key inequalities (1.6) and (1.8) from Zhang's inequality, paying a penalty on the constant. We use similar ideas to those appearing in [24] which prove the Polya-Szegö rearrangement inequality from the isoperimetric inequality.

Proposition 3.1. Let $1 \leq p<\infty$. For all $f \in W^{1, p}\left(\mathbb{R}^{n}\right)$,

$$
\mathcal{E}_{p}\left(f^{\circ}\right) \leq \frac{I_{p}}{I_{1}} \mathcal{E}_{p}(f) \quad \text { and } \quad \mathcal{E}_{p}^{+}\left(f^{\circ}\right) \leq \frac{I_{p}}{I_{1}} \mathcal{E}_{p}^{+}(f) .
$$

Remark 3.2. It is well known that $C_{1} \sqrt{p} \leq \frac{I_{p}}{I_{1}} \leq C_{2} \sqrt{p}$ with $C_{1}, C_{2}$ absolute constants.

Proof. Suppose $f$ is a $\mathcal{C}^{1}$ function of compact support. Let $\Phi(t)$ represent either $|t|$ or $\max \{t, 0\}$. By Sard's theorem, for almost all $t>0$ the level set $\{|f| \geq t\}$ is compact with $\mathcal{C}^{1}$ boundary $\{|f|=t\}$ and $\nabla f(x) \neq 0, \forall x \in\{|f|=t\}$. By Federer's co-area formula,

$$
\int_{\mathbb{R}^{n}} \Phi(\langle\nabla f(x), u\rangle)^{p} d x=\int_{0}^{\infty}\left(\int_{\{|f|=t\}} \Phi(\langle\nabla f(x), u\rangle)^{p} d \mu(x)\right) d t
$$


where, for almost every $t>0, d \mu(x)=\frac{d H_{n-1}(x)}{|\nabla f(x)|}, d H_{n-1}(x)$ being the corresponding Hausdorff measure on $\{|f|=t\}$. Next we use Jensen's inequality,

$$
\begin{aligned}
\int_{\mathbb{R}^{n}} \Phi(\langle\nabla f(x), u\rangle)^{p} d x & \geq \int_{0}^{\infty}\left(\int_{\{|f|=t\}} \Phi(\langle\nabla f(x), u\rangle) \frac{d \mu(x)}{\int_{\{|f|=t\}} d \mu(x)}\right)^{p}\left(\int_{\{|f|=t\}} d \mu(x)\right) d t \\
& =\int_{0}^{\infty}\left(\int_{\{|f|=t\}} \Phi(\langle\nabla f(x), u\rangle) d \mu(x)\right)^{p}\left(\int_{\{|f|=t\}} d \mu(x)\right)^{1-p} d t .
\end{aligned}
$$

Denote $M(t)=\int_{\{|f| \geq t\}} d x$. For almost every $t>0$, another use of the co-area formula yields

$$
\int_{\{|f|=t\}} d \mu(x)=\left(-\int_{t}^{\infty}\left(\int_{\{|f|=s\}} \frac{d H_{n-1}(x)}{|\nabla f(x)|}\right) d s\right)^{\prime}=-M^{\prime}(t)=\left|M^{\prime}(t)\right|
$$

and so

$$
\begin{aligned}
& \left(\int_{S^{n-1}}\left(\int_{\mathbb{R}^{n}} \Phi(\langle\nabla f(x), u\rangle)^{p} d x\right)^{-n / p} d u\right)^{-p / n} \\
& \geq\left(\int_{S^{n-1}}\left(\int_{0}^{\infty}\left(\int_{\{|f|=t\}} \Phi(\langle\nabla f(x), u\rangle) d \mu(x)\right)^{p}\left|M^{\prime}(t)\right|^{1-p} d t\right)^{-n / p} d u\right)^{-p / n} .
\end{aligned}
$$

Use Minkowski's integral inequality to bound the previous formula from below:

$$
\begin{aligned}
& \geq \int_{0}^{\infty}\left(\int_{S^{n-1}}\left(\int_{\{|f|=t\}} \Phi(\langle\nabla f(x), u\rangle) d \mu(x)\right)^{-n} d u\right)^{-p / n}\left|M^{\prime}(t)\right|^{1-p} d t \\
& =\int_{0}^{\infty}\left(\int_{S^{n-1}}\left(\int_{\{|f|=t\}} \Phi(\langle\nu(x), u\rangle) d H_{n-1}(x)\right)^{-n} d u\right)^{-p / n}\left|M^{\prime}(t)\right|^{1-p} d t,
\end{aligned}
$$

where for a.e. $t>0, \nu(x)$ is the outer normal vector to $\{|f|=t\}$ (w.r.t. $\{|f| \geq t\}$ ) at the point $x$.

For every "good" $t>0$ from Sard's theorem, one can easily see, 32, that the linear functional $g \in \mathcal{C}\left(S^{n-1}\right) \rightarrow \int_{\{|f|=t\}} g(\nu(x)) d H_{n-1}(x)$ can be represented by a finite measure $d \mu_{t}$ on $S^{n-1}$. That is,

$$
\int_{S^{n-1}} g(v) d \mu_{t}(v)=\int_{\{|f|=t\}} g(\nu(x)) d H_{n-1}(x) \quad \forall g \in \mathcal{C}\left(S^{n-1}\right) .
$$

Recall (see for instance 27]) that every convex body $K \subset \mathbb{R}^{n}$ determines a surface area measure on $S^{n-1}$ denoted by $S_{K}$.

It is also proved in [32] that, by the Minkowski existence theorem (see [27]), there exists a unique up to translations convex body $K_{t}$ in $\mathbb{R}^{n}$ whose surface area measure $S_{K_{t}}$ is $\mu_{t}$. For this reason $\mu_{t}$ is also called the surface area measure of (the compact set) $\{|f| \geq t\}$. 
Let $\Pi K_{t}$ be the projection body associated to $K_{t}$, i.e. the convex body defined by its support function as

$$
h\left(\Pi K_{t}, u\right)=\frac{1}{2} \int_{S^{n-1}}|\langle v, u\rangle| d S_{K_{t}}(v)=\left|P_{u \perp}\left(K_{t}\right)\right|_{n-1}, \quad u \in S^{n-1} .
$$

Let $\Pi^{*} K_{t}$ be the polar projection body of $K_{t}$. Its volume is

$$
\begin{aligned}
2^{-n}\left|\Pi^{*} K_{t}\right|_{n} & =\int_{S^{n-1}} h\left(\Pi K_{t}, u\right)^{-n} d u=\int_{S^{n-1}}\left(\int_{S^{n-1}}|\langle u, v\rangle| d S_{K_{t}}(v)\right)^{-n} d u \\
& =\int_{S^{n-1}}\left(\int_{\{|f|=t\}}|\langle\nu(x), u\rangle| d H_{n-1}(x)\right)^{-n} d u .
\end{aligned}
$$

Finally, Petty's projection inequality (1.5) and the fact proved in [32, $M(t)=$ $|\{|f| \geq t\}|_{n} \leq\left|K_{t}\right|_{n}$, show that for almost all $t>0$,

$$
\left(\int_{S^{n-1}}\left(\int_{\{|f|=t\}}|\langle\nu(x), u\rangle| d H_{n-1}(x)\right)^{-n} d u\right)^{-1 / n} \geq 2 \frac{\omega_{n-1}}{\omega_{n}^{1-1 / n}} M(t)^{\frac{(n-1)}{n}} .
$$

Consider the case $\Phi(t)=|t| . \quad M$ and $f^{*}$ are differentiable (except, possibly, on some set $N$ of zero measure) and $M=\left(f^{*}\right)^{-1}$ on the set $\left(f^{*}\right)^{-1}((0, \infty) \backslash N)$; therefore

$$
\begin{aligned}
\left(\int_{S^{n-1}}\left\|D_{u} f\right\|_{p}^{-n} d u\right)^{-p / n} & \geq 2^{p}\left(\frac{\omega_{n-1}}{\omega_{n}^{1-1 / n}}\right)^{p} \int_{0}^{\infty} M(t)^{\frac{(n-1) p}{n}}\left|M^{\prime}(t)\right|^{1-p} d t \\
& =\left(\frac{2 \omega_{n-1}}{\omega_{n}^{1-1 / n}}\right)^{p} \int_{0}^{\infty} s^{\frac{p(n-1)}{n}}\left|f^{* \prime}(s)\right|^{p} d s .
\end{aligned}
$$

In the other case, $\Phi(t)=\max \{t, 0\}$, since $\int_{S^{n-1}}\langle u, v\rangle d \mu_{t}(v)=0$ we have

$$
h\left(\Pi K_{t}, u\right)=\frac{1}{2} \int_{S^{n-1}}|\langle v, u\rangle| d \mu_{t}(v)=\int_{S^{n-1}}\langle v, u\rangle_{+} d \mu_{t}(v) .
$$

Hence

$$
\left(\int_{S^{n-1}}\left\|D_{u}^{+} f\right\|_{p}^{-n} d u\right)^{-p / n} \geq\left(\frac{\omega_{n-1}}{\omega_{n}^{1-1 / n}}\right)^{p} \int_{0}^{\infty} s^{\frac{p(n-1)}{n}}\left|f^{* \prime}(s)\right|^{p} d s .
$$

Finally we recall (2.1) and analogously

$$
\left(\int_{S^{n-1}}\left\|D_{u} f^{\circ}\right\|_{p}^{-n} d u\right)^{-p / n}=\left(n \omega_{n}^{1 / n}\right)^{p}\left(\int_{0}^{\infty} s^{\frac{(n-1) p}{n}}\left|f^{* \prime}(s)\right|^{p} d s\right) I_{p}^{p} .
$$

Therefore

$$
\mathcal{E}_{p}\left(f^{\circ}\right) \leq \frac{n \omega_{n}}{2 \omega_{n-1}} I_{p} \mathcal{E}_{p}(f)=\frac{I_{p}}{I_{1}} \mathcal{E}_{p}(f) \quad \text { and } \quad \mathcal{E}_{p}^{+}\left(f^{\circ}\right) \leq \frac{n \omega_{n}}{2 \omega_{n-1}} I_{p} \mathcal{E}_{p}^{+}(f)=\frac{I_{p}}{I_{1}} \mathcal{E}_{p}^{+}(f) \text {. }
$$

\section{ACKNOWLEDGEMENT}

The authors thank the referee for useful comments that helped to improve the presentation of the manuscript. 


\section{REFERENCES}

1. T. Aubin, Problèmes isopérimétriques et espaces de Sobolev, J. Differential Geom. 11 (1976), 573-598. MR0448404 (56:6711)

2. S. Bobkov and M. Ledoux, From Brunn-Minkowski to sharp Sobolev inequalities, Ann. Mat. Pura Appl.(4) 187 (2008), 369-384. MR2393140 (2010i:46052)

3. J. Bastero, M. Milman and F.J. Ruiz, A note on $L(\infty, q)$ spaces and Sobolev embeddings, Indiana U. Math. J. 52 (2003), 1215-1229. MR2010324 (2004h:46025)

4. H. Brézis and S. Wainger, A note on limiting cases of Sobolev embeddings and convolution inequalities, Comm. Partial Diff. Eq. 5 (1980), 773-789. MR579997 (81k:46028)

5. I. Chavel, Isoperimetric Inequalities. Differential Geometric and Analytic Perspectives in Mathematical Physics, Cambridge Tracts in Mathematics 145, Cambridge Univ. Press, 2001. MR:1849187(2002h:58040)

6. A. Cianchi, E. Lutwak, D. Yang and G. Zhang, Affine Moser-Trudinger and Morrey-Sobolev inequalities, Calc. Var. Partial Differ. Equ. 36 (2009), 419-436. MR2551138(2010h:46041)

7. D. Cordero-Erausquin, B. Nazaret, and C. Villani, A mass-transportation approach to sharp Sobolev and Gagliardo-Nirenberg inequalities, Adv. Math. 182 (2004), 307-332. MR2032031 (2005b:26023)

8. H. Federer and W. H. Fleming, Normal integral currents. Ann. of Math. (2) 72 (1960), 458520. MR0123260 (23:A588)

9. C. Haberl and F.E. Schuster, Asymmetric affine $L_{p}$ Sobolev inequalities, J. Funct. Anal. 257 (2009), 641-658. MR2530600 (2010j:46068)

10. C. Haberl and F.E. Schuster, General $L_{p}$ affine isoperimetric inequalties, J. Differential Geom. 83 (2009), 1-26. MR2545028 (2010j:52026)

11. C. Haberl, F.E. Schuster and J. Xiao, An asymmetric affine Pólya-Szegö principle, preprint.

12. K. Hansson, Imbedding theorems of Sobolev type in potential theory, Math. Scand. 45 (1979), 77-102. MR567435 (81j:31007)

13. S. Kesavan, Symmetrization $\mathscr{E}$ Applications. Series in Analysis, vol. 3. World Scientific Publ., Hackensack, NJ, 2006. MR2238193(2008a:35005)

14. V. Kolyada, Rearrangements of functions and embedding theorems, Russ. Math. Surveys Eq. 44 (1989), 73-117. MR 1040269 (91i:46029)

15. E. Lutwak, The Brunn-Minkowski-Firey Theory I: Mixed volumes and the Minkowski problem. J. Differential Geom. 38 (1993), 131-150. MR1231704 (94g:52008)

16. E. Lutwak, D. Yang and G. Zhang, On $L_{p}$ affine isoperimetric inequalities, J. Differential Geom. 56 (2000), 111-132. MR1863023 (2002h:52011)

17. E. Lutwak, D. Yang and G. Zhang, Sharp affine $L_{p}$ Sobolev inequalities, J. Differential Geom. 62 (2002), 17-38. MR1987375 (2004d:46039)

18. W. G. Maz'ja, Classes of domains and embedding theorems for functional spaces, Soviet Math. Dokl. 1 (1961), 882-885. MR0126152 (23:A3448)

19. W. G. Maz'ja, Sobolev spaces, Springer Series in Soviet Mathematics 145, Springer-Verlag, Berlin, 1985. MR817985 (87g:46056)

20. J. Malý and L. Pick, An elementary proof of sharp Sobolev embeddings, Proc. Amer. Math. Soc. 13 (2002), 555-563. MR1862137 (2002j:46042)

21. J. Martin and M. Milman, Pointwise symmetrization inequalities for Sobolev functions and applications, Adv. Math. 225 (2010), 121-199. MR2669351

22. J. Martin, M. Milman, and E. Pustylnik, Sobolev inequalities: symmetrization and selfimprovement via truncation, J. Funct. Anal. 252 (2) (2007), 677-695. MR2360932 (2009a:46059)

23. J. Moser, A sharp form of an inequality by Trudinger, Indiana Univ. Math. J. 20 (1971), 1077-1092. MR0301504 (46:662)

24. J. Mossino, Inégalités Isopérimétriques et Applications en Physique, Travaux en Cours, Hermann, Paris, 1984. MR733257 (85k:49002)

25. C. M. Petty, Centroid surfaces, Pacific J. Math. 11 (1961), 1535-1547. MR0133733 (24:A3558)

26. G. Pólya and G. Szegö, Isoperimetric Inequalities in Mathematical Physics, Ann. Math. Stu. 27, Princeton University Press, Princeton, NJ, 1951. MR0043486(13:270d)

27. R. Schneider, Convex Bodies: The Brunn-Minkowski Theory, Cambridge University Press, Cambridge, 1993. MR 1216521 (94d:52007) 
28. G. Talenti, Best constants in Sobolev inequality, Ann. Math. Pura Appl. 110 (1976), 353-372. MR0463908(57:3846)

29. G. Talenti, Inequalities in rearrangement invariant function spaces, Nonlinear Analysis, Function Spaces and Applications 5, Prometheus, Prague, 177-230, 1994. MR1322313(96a:46062)

30. L. Tartar, Imbedding theorems of Sobolev spaces into Lorentz spaces, Boll. Unione Mat. Ital. Sez. B Artic. Ric. Mat. 8, 1 (1998), 479-500. MR.1662313 (99k:46060)

31. N.S. Trudinger, On imbeddings into Orlicz spaces and some applications, J. Math. Mech. 17 (1967), 473-483. MR0216286(35:7121)

32. G. Zhang, The affine Sobolev inequality, J. Differential Geom. 53 (1999), 183-202. MR.1776095 (2001m:53136)

Departamento de Matemáticas, Universidad de Zaragoza, 50009 Zaragoza, Spain

E-mail address: daalonso@unizar.es

Departamento de Matemáticas, Universidad de Zaragoza, 50009 Zaragoza, Spain

E-mail address: bastero@unizar.es

Departamento de Matemáticas, Universidad de Zaragoza, 50009 Zaragoza, Spain

E-mail address: bernues@unizar.es 